\title{
Postprandial effects of supplementing a moderate-fat meal with long-chain n-3 PUFA on measures of arterial stiffness
}

\author{
S. Lockyer, M. F.-F. Chong, C. J. Saunders, A. L. Carvalho-Wells and J. A. Lovegrove \\ Hugh Sinclair Unit of Human Nutrition, Department of Food Biosciences, University of Reading, Reading, UK
}

It is well established that the consumption of long-chain (LC) $n-3$ PUFA, EPA and DHA, found in fish oils are cardio-protective. Arterial stiffening is a manifestation of vascular dysfunction and is recognised as a biomarker for $\mathrm{CVD}^{(1)}$. There are limited and inconsistent data on the influence of LC $n$-3 PUFA on arterial stiffness in the postprandial state ${ }^{(2,3)}$. The aim of the present study was to investigate the effects of a moderate-fat meal enriched with LC $n$-3 PUFA on arterial stiffness determined by two different techniques, digital volume pulse analysis (DVP) and pulse wave analysis (PWA), both of which have been described previously ${ }^{(4)}$. The DVP was obtained by photoplethysmography using the PulseTrace system (Micro Medical, Chatham Maritime, Kent, UK) to calculate stiffness index (SI). PWA was performed by applanation tonometry at the radial artery using the SphygmoCor (ScanMed Medical, Moreton-in-Marsh, Glos., UK) to calculate augmentation index (AIx).

Twenty-five healthy subjects (twelve males, thirteen females; age 47 (SD 4) years, BMI 23.4 (SD 0.7) kg/m² received either a control (CT) meal (30 g mixed fat; fatty acid profile representative of the UK diet) or an LC $n-3$ PUFA-rich meal ( $23 \mathrm{~g}$ mixed fat, $6.7 \mathrm{~g}$ fish oil, which provided $2.0 \mathrm{~g}$ EPA and $2.7 \mathrm{~g}$ DHA) on two occasions in a random order. DVP and PWA measurements were taken at baseline and 30, 60, 90, 120, 180 and 240 min after meal consumption to derive SI and AIx respectively. Blood samples were taken at baseline and 120 and 240 min after meal consumption and were analysed for glucose, TAG, insulin and NEFA.
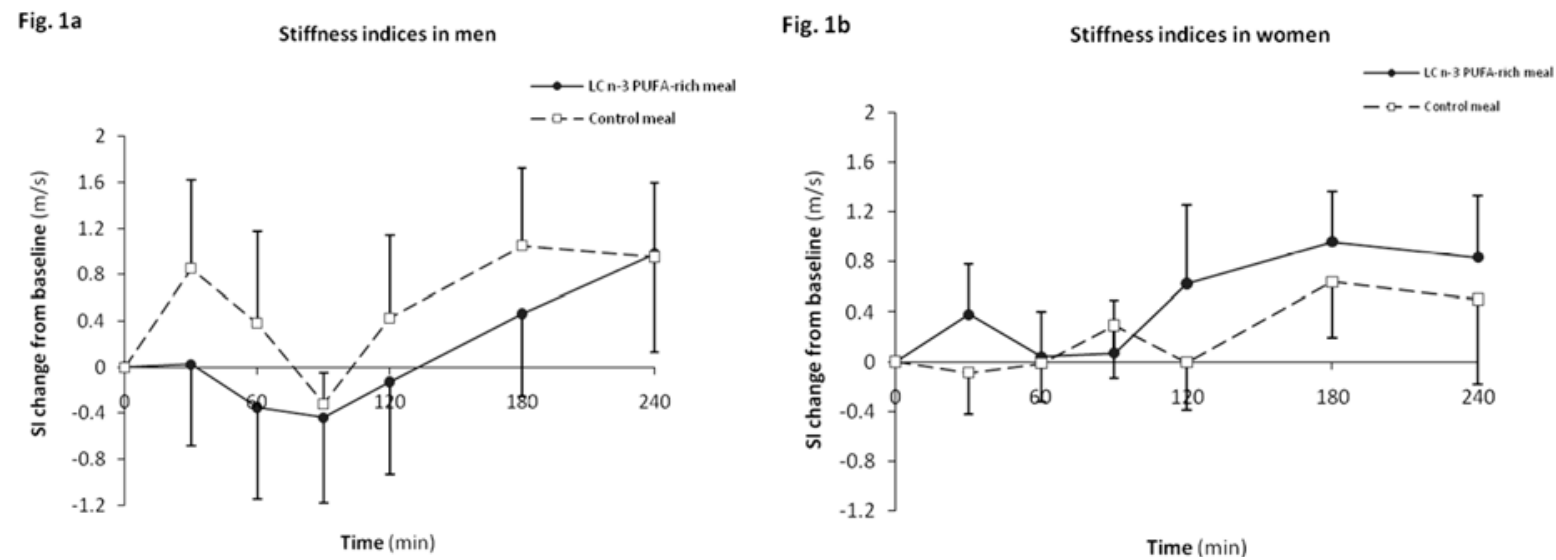

Fig. 2 Augmentation indices in all subjects

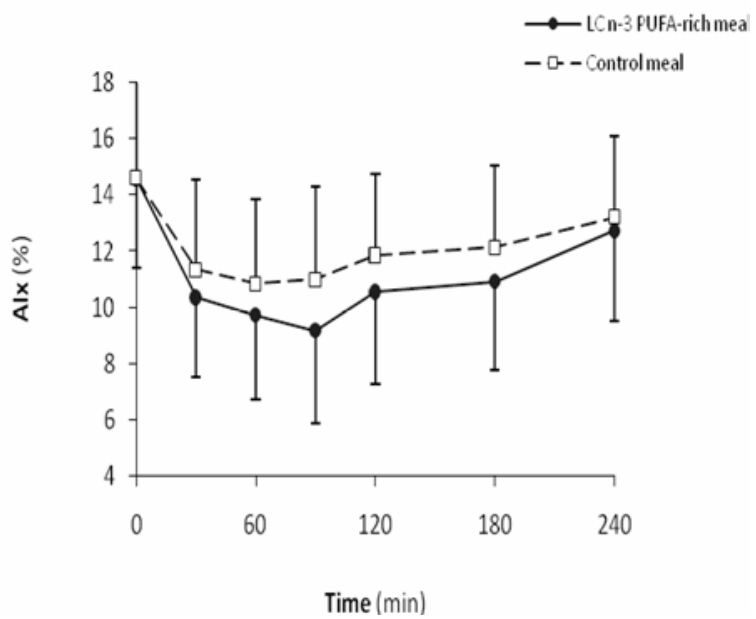

A significant gender $\times$ treatment interaction $(P=0.011)$ was observed for SI. In men the LC $n$-3 PUFA-rich meal had an attenuating effect on SI compared with the CT meal (Fig. 1(a); $P=0.0011)$. In women SI were close to or above baseline after both meals and only rose above baseline at $180 \mathrm{~min}$ (Fig. 1(b); $P=0.74)$. There was a trend for AIx to be consistently lower after the LC $n$-3 PUFA-rich meal compared with the CT meal (Fig. 2; $P=0.099)$. A significant treatment effect $(P=0.036)$ was seen for NEFA concentrations (data not shown). Changes in insulin, glucose and TAG concentrations did not differ between the two meals.

The data provides evidence that acute LC $n-3$ PUFA-rich meal consumption improves postprandial arterial stiffness measured by DVP in men but not in women when compared with a CT meal.

1. Malik AR, Kondragunta V \& Kullo IJ (2008) Hypertension 51, 1512 1518.

2. Khan F, Elherik K, Bolton-Smith C et al. (2003) Cardiovasc Res 59, 955-962.

3. Hall WL, Saunders KA, Sanders TAB et al. (2008) J Nutr 138, 287 229.

4. Lane HA, Smith JC \& Davies JS (2006) Vasc Health Risk Manag 2, $19-30$. 